DOI: 10.4312/an.52.1-2.153-166

\title{
“Tell him anyway": Zu Uwe Timms Roman Ikarien
}

\author{
Špela Virant
}

\section{ABSTRACT}

Der Roman Ikarien (2017) von Uwe Tim ist in Deutschland der Nachkriegszeit verortet, wo Wagner einem jungen amerikanischen Offizier die Lebensgeschichte des Eugenikers Alfred Ploetz erzählt. Obwohl Timm einen historischen Stoff verarbeitet, werden im Roman aktuelle und philosophische Themen angesprochen. Timm verfolgt dabei Ideen, Denkmuster und Denkfehler, die zu Gewalt, Rassismus und Genozid führen können. Er entwickelt aber auch literarische Gegenbilder dazu. Da sich Timm bereits seit Jahrzehnten in seinen Werken mit diesen Themen beschäftigt, wird der Roman Ikarien im Kontext seines Euvres und der Geschichte von Utopien gestellt, auf die schon der Romantitel verweist. ${ }^{1}$

Schlüsselwörter: zeitgenössische deutschsprachige Literatur, Uwe Timm, Ikarien, Morenga, Eugenik, Utopie

1 Der Beitrag ist im Rahmen des Forschungsprogramms Interkulturelle literaturwissenschaftliche Studien (Nr. P6-0265) entstanden, das von der Slowenischen Forschungsagentur aus öffentlichen Mitteln finanziert wird. 
„Wagner: Verzeiht! Es ist ein groß Ergetzen,

Sich in den Geist der Zeiten zu versetzen,

$\mathrm{Zu}$ schauen wie vor uns ein weiser Mann gedacht,

Und wie wir's dann zuletzt so herrlich weit gebracht."

(Goethe 2007: 373)

Es ist ein anderer Wagner, der in Uwe Timms Roman Ikarien (2017) über die Gedanken des Wissenschaftlers, des Eugenikers, Alfred Ploetz erzählt und mit seiner Erzählung zeigt, dass Wissenschaft nicht mit Weisheit verwechselt werden darf. Sein Zuhörer ist Michael Hansen, ein junger amerikanischer Offizier deutscher Herkunft, der ihn in den ersten Wochen nach dem Ende des Zweiten Weltkriegs in den Trümmern der zerstörten deutschen Städte ausfindig macht und die Aufgabe bekommt, ihn zu verhören. Sicherlich war es auch ein Verdienst von Wissenschaftlern wie Alfred Ploetz, dass die Nationalsozialisten es so erschreckend „weit gebracht" hatten, doch ist Timms Ikarien weit mehr als nur ein historischer und biographischer Roman, ${ }^{2}$ denn er stellt, im Subtext, die Frage, wie weit wir es heute gebracht haben und wie weit wir es noch bringen werden, wenn wir so weitermachen. Wie andere wichtige Kunstwerke, lässt auch dieser Roman sich nicht mit einer simplen Etikettierung erfassen. Neben Elementen des historischen, biographischen, sozialkritischen und politischen Romans, die sich dem Text nicht absprechen lassen, ist es auch oder gar vor allem - so die These, die im Folgenden ausgeführt werden soll - ein philosophischer Roman, da er spezifische Denkweisen untersucht, nach ihren Quellen forscht und ihre Folgen verbildlicht. Um diese Aspekte besser darzustellen, soll Ikarien zunächst im Kontext von Timms Euvre verortet werden, denn die Grundfragen, die dieser Roman stellt, begleiten Timm, wie Christine Setzwein berichtet, bereits seit Jahrzehnten:

Die Idee zu „Ikarien“ hatte Timm schon seit 1978, als er „Morenga“ abgeschlossen hatte. Sein Schwiegervater, der älteste Sohn von Alfred Ploetz, habe viel erzählt von seinem Vater. Und die Frage, die Timm schon seit „Morenga“ umtrieb, stellte sich nun auch bei der Beschäftigung mit dem Eugeniker: „Woher kommt dieser Vernichtungswille? Und wie kann ein so begabter und idealistisch friedfertiger Mensch so abdriften ins rassistische Denken?" (Setzwein 2018)

Der Roman Morenga, 1978 erschienen, gilt als erster deutschsprachiger postkolonialer Roman, der die zögerliche Aufarbeitung des wilhelmini-

2 Vgl. z.B. Rainer Moritz (2017), der dem Roman, weil er sich dem ihm zugeschriebenen Genre eines biographischen Romans nicht fügt, eine „Masse an Fakten“ und „Stofffülle“ vorwirft. Carsten Otte (2017) schätzt zwar das Buch, bezeichnet es jedoch als „ein erschreckend aktuelles Geschichtsbuch" und fokussiert sich vor allem auf die historischen Aspekte des Romans. 
schen Kolonialismus einleitete und dessen Qualität und Bedeutung von der Literaturwissenschaft erst relativ spät anerkannt wurde. ${ }^{3}$ Der Roman, der verschiedene Geschichten und Diskursformen collagiert, ${ }^{4}$ erzählt die Geschichte des Veterinärs Gottschalk und durch sie die Geschichte des Aufstands der Nama und der Herero gegen die deutschen Besatzer in Deutsch-Südwestafrika in den Jahren zwischen 1904 und 1907, der mit einem Genozid an ihnen endete. Schon in diesem Roman greift Timm weit zurück ins 19. Jahrhundert und erzählt skurrile Geschichten über einzelne Abenteurer, die das Land bereisten, missionierten, geschäftlich ausbeuteten oder fotografierten - noch vor der Gründung der Deutschen Kolonialgesellschaft und den Verbrechen der Schutztruppe, die Gottschalk in Taten- und Sprachlosigkeit versetzen. Doch bevor es soweit ist, lässt Timm ihn in sein Tagebuch die Fragen schreiben, die sich angesichts eines jeden Völkermords seit Beginn der Neuzeit von neuem stellen:

Wie kommt es zur Tötung? Wie können Menschen andere erschießen oder erhängen? Und wie können andere zusehen wie auf einem Jahrmarkt? Was erzeugt diese Teilnahmslosigkeit und darunter diesen fürchterlichen Haß? Vielleicht ist etwas in ihnen, was ihnen selbst hassenswert ist, ein Teil ungelebten Lebens. Was tötet das ab, das Mitfühlen? (Timm 2010: 388)

Der Roman Morenga versucht von Anfang an mögliche Antworten auf diese Fragen zu finden, noch bevor Gottschalk sie expliziert, ungeachtet der Gefahr, dass es womöglich keine Antworten gibt. Die Fiktion ist hier der Raum, um mögliche Antworten durchzuspielen.

Die erste mögliche Antwort bezieht sich auf die finanziellen Interessen, die zwar die Gründung der Kolonialgesellschaften erklären mögen, im Individuellen jedoch unzureichend erscheinen, wie die Geschichte von Klügge es zeigt, dem Unternehmer, der mit einem riesengroßen Fass Brandwein durchs Land zieht: „Er

3 Manfred Durzak vergleicht Timms Roman Morenga mit dem 25 Jahre später erschienenen Roman Herero (2003) von Gerhard Seyfried und stellt fest: „Herero behauptet sich höchstens als monumentale Fussnote im Vergleich zu Morenga." (Durzak 2007: 47)

4 Elke Frederiksen beschreibt seine Erzähltechnik sehr genau: „The encounter of these very different cultures (German and African) finds expression in an unusual narrative process, which presents us with a multi-perspective montage of fiction and nonfiction. Documentary reports by the General Staff, telegrams, letters, quotes from colonial memoirs share space with fictional diary entries, geographical and ethnic reports, as well as tales, legends and myths (of the Namas). This montage technique skillfully integrates imagined and authentic text components. Historical documents (identified by precise bibliographical notations) and fictitious elements, nevertheless, blend to such an extent that they cannot always be differentiated. The result is a historical novel, which - while utilizing a de-centered narrative - rewrites the tradition of German colonial novels and succeeds in breaking open hierarchical structures by confronting the reader with widely differing perspectives." (Frederiksen 2013: 266) 
wollte Geld verdienen, und zwar viel. Das Merkwürdige war, daß er dabei nicht daran dachte, was er mit dem Geld einmal machen würde." (Timm 2010: 188) Auch später, als er dann doch Vorstellungen entwickelt, was er machen könnte, kann er „sich selbst mit den genannten Plänen nicht in Verbindung“ (214) bringen und er wird sie auch nie realisieren. So unreflektiert, wie Klügge das Gebot des Kapitalismus, unbedingt Profit zu erwirtschaften, übernimmt, übernehmen andere das alttestamentarische Gebot, sich die Erde untertan zu machen (1.Mose 1,28) - "Jonker und seine Männer bezogen das auf die Weidegebiete der Herero“ (Timm 2010: 199) -, oder die militaristische Prämisse, „daß die Hottentotten die Todfeinde waren“ (375). Als Gottschalk auf den Fragen nach den Ursachen insistiert, wird er abgewiesen, es seien „Sachzwänge, unbeeinflußbar vom einzelnen“ (ebd.). Diese Gebote werden zwar von Gottschalk und einigen anderen Figuren zeitweise angezweifelt, von den meisten jedoch unhinterfragt befolgt. Das Befolgen dieser Gebote, durch welches einerseits die Illusion eines stabil geordneten Lebens und überlegener Zivilisiertheit genährt wird, führt andererseits zum Verlust der Lebensfreude und dem Gefühl eines „ungelebten Lebens“. Wenn die derart „zivilisierten“ oder selbstverstümmelten Kolonialisten auf Menschen treffen, bei denen sie die Lebenslust zu beobachten meinen, die sie sich selbst versagen, entwickeln sie Hass aus einer Art Neid. So könnte die suggerierte sozialpsychologische Erklärung zusammengefasst werden. Die erwähnten Gebote der „Zivilisation“, in denen sich kapitalistische Ideologie, Militarismus und religiöse Dogmen verschränken, wurden seit der frühen Neuzeit entwickelt und eingesetzt, um den Kolonialismus und Paternalismus zu rechtfertigen. Sie prägen zum Teil bis heute die Beziehungen der sogenannten Entwickelten zum Rest der Welt, obwohl sie von post- und dekolonialen Theorien bereits dekonstruiert wurden. Doch in der Figur Gottschalks kündigt sich eine weitere Frage an, die sich in den Diskussionen um die Nach-Postmoderne zwar oft stellt, jedoch mit Vorsicht behandelt wird: die Frage nach der Zukunft, nach neuen Denkweisen, Visionen und Utopien.

Gottschalk wehrt den Vorwurf, er habe mit seiner Arbeit die Kolonialherren bei dem Völkermord unterstützt, mit dem Verweis auf eine Zukunft ab, in der seine Erkenntnisse „allen Menschen in diesem Lande zugute kommen würden. Irgendwann. Eben. Aber wann war das, dieses Irgendwann?" (Timm 2010: 386) Als Wissenschaftler und Erfinder vertritt er hier die Idee eines stätigen Fortschritts in eine offene Zukunft, der verallgemeinernd „allen Menschen“ Gutes bringen wird. Ebenfalls verlangt er nach neuen Denkweisen: „Man muß etwas Neues denken, etwas ganz anderes zu dem, wie man selbst lebt.“ (376) Es ist eine Forderung nach Utopie, die sich in Gottschalks Worten artikuliert, nach Ideen, die im Leben, wie es hier und jetzt gelebt wird, keinen Platz haben. Doch es ist ihm nicht möglich, sich für den politischen Aktivismus und revolutionäre 
Visionen zu begeistern, die den Einzelnen ausblenden. In Gesprächen mit dem zukunftsorientierten und Technik-begeisterten Leutnant Elschner erklärt er wiederholt seine Ansichten: „Elschner hatte einmal gelegentlich zu Gottschalk gesagt: Der einzelne ist nichts. Der einzelne ist alles, hatte Gottschalk geantwortet.“ (386) „Dagegen sagte Gottschalk, es komme gerade auf den einzelnen an, auf ihn ganz allein." (375) Bald danach kündigt er den Dienst bei der Schutztruppe, kehrt nach Deutschland zurück, wird Professor und Ballonfahrer.

Gottschalks Betonung des Einzelnen lässt eine innere Logik erahnen, nach der es plausibel ist, dass er sich von allen Welteroberungs- und Weltrettungsversuchen abwendet und seine eigene individuelle Entwicklung verfolgt. In diesem Sinne erscheint auch die Interpretation von Stefan Neuhaus folgerichtig:

Ohne dass dem Leser Innensicht in die Figur gewährt wird, lässt sich nachvollziehen, wie Gottschalks Position erodiert, seine bisherigen Vorstellungen nach und nach außer Kraft gesetzt werden. Daran ist nichts Negatives, im Gegenteil. Es stellt sich heraus, dass dies die Herausbildung einer eigenen, individuellen Identität erst ermöglicht. Dafür steht der Schluss, die Ballonfahrt Gottschalks nach seiner Rückkehr aus Afrika: Er wird hier symbolisch in Freiheit gesetzt. (Neuhaus 2004: 88-89)

Doch was hier aus der Perspektive einer konsistenten Figurenentwicklung im Kontext des Romans wie auch im Kontext der deutschsprachigen Literatur der 1970er Jahre mit ihrem deklarierten Rückzug ins Private überzeugend erscheint, reicht nicht aus für eine Auslegung des gesamten Romans und schon gar nicht für die Verdeutlichung von Timms poetologischer Position. Der Text ist zu komplex und die gestellten Fragen zu schwerwiegend, als dass sie sich in einem Ballon, einer resignativen Selbstverwirklichung, ,aufheben“ ließen. Wenn Gottschalk dem Genozid nichts als Ballonfahren entgegensetzen kann, gilt das nicht für Timms Poetik. Julia Schöll untersucht Timms poetologische Schriften und stellt fest: „Von der Position, dass Literatur die Welt verändert - was eine gewisse Machtposition des Autors impliziert -, rückt Uwe Timm in späteren poetologischen Entwürfen explizit ab.“ (Schöll 2012: 30) Die spätere Position fasst sie zusammen: „Der Mensch, der die Welt verändern möchte, muss zunächst seine Wahrnehmung verändern; darin besteht der utopische und humanistische gesellschaftliche Beitrag, den Literatur zu leisten imstande ist." (Schöll 2012: 32)

Gottschalks Ballonfahrt ermöglicht einen Perspektivwechsel, gewährt einen neuen Ausblick und eine veränderte Wahrnehmung der Welt. Während sich Leutnant Elschner für Aeroplane begeistert, die bereits einige Jahre später im Ersten Weltkrieg einen entscheidenden militärischen Vorteil durch Luftaufklärung sichern werden, ist die Ballonfahrt ein friedlicher Versuch, durch 
Distanz und Übersicht, Einblick und Erkenntnis zu gewinnen. Ikarien, die vier Jahrzehnte gereifte Fortsetzung der Thematik von Morenga, ist in gewisser Weise ein poetischer - aber keineswegs utopischer - Bericht über die so gewonnenen Erkenntnisse.

Wenn auch Morenga auf die Frage, warum potentiell genozidale Systeme fortbestehen können, mit dem Verweis auf die durch kollektive Frustrationen entwickelten Verhaltensweisen einerseits und das Zusammenspiel partieller, individueller Interessen andererseits mögliche Antworten anbietet, wird die Frage nach den Ursachen des Scheiterns bereits angedachter Alternativen, nach denen sich Gottschalk umsieht, erst in Ikarien gestellt.

Es liegt eine erschütternde Klarheit und Plausibilität in der Art, wie Uwe Timm die Geschichte einer Idee, deren Träger die Romanfigur Alfred Ploetz ist, erzählt. Der junge Ploetz begeistert sich, auf seiner Suche nach sozialer Gerechtigkeit, über marxistische Ideen, besucht in den USA die von Étienne Cabet gegründete Gemeinschaft der Ikarier, bemerkt ihr Scheitern und kommt im Grübeln über die Gründe ihres Scheiterns zu dem Schluss, dass das gesellschaftliche Konzept der égalité zwar gut sei, die Menschen davon aber wegen ihrer unterschiedlichen körperlichen und geistigen Voraussetzungen nicht gleichermaßen profitieren können, weshalb es notwendig sei, sie, die Menschen, der Idee anzupassen und sie, durch das Projekt der Eugenik, biologisch auf einer höheren Entwicklungsstufe einander möglichst anzugleichen. Ploetz hat Anlagen für eine tragische Figur, die Gutes will und Böses tut. Die Figur regt zum Nachdenken über den tragischen Irrtum an, also über den Fehler, der eine schöne Utopie in grausame Realität umschlagen lässt. Wird dabei die Geschichte der Utopien beachtet, so zeigt sich die individuelle Problematik der Figur Ploetz als Konsequenz von einer ganzen Reihe von Verschiebungen und Fehlentwicklungen im europäischen neuzeitlichen Denken über eine ideale Gesellschaftsordnung.

Der Begriff Utopie, der von Thomas Morus geprägt wurde, ist, so Thomas Schölderle in seiner übersichtlichen Studie Geschichte der Utopie (2012), „die Bezeichnung für ein literarisches Genre, später ein allgemein gebräuchlicher Begriff, letztlich ein vieldeutiges Schlagwort." (Schölderle 2012: 9)5 Als das wichtigste Element von Utopien bezeichnet er „die Intention unmittelbarer Sozialkritik.“ (12) Der Altphilologe Marko Marinčič, der utopische Elemente in antiken literarischen Vorläufen dieser Gattung untersucht, bemerkt, dass sich alle Utopien im gleichen Maße mit dem Propagieren politischer Ideen befassen

5 Schölderle fast einleitend zusammen, dass sich in der bisherigen Utopieforschung „ein klassischer, ein sozialpsychologischer sowie ein totalitarismustheoretischer Utopiebegriff" (Schölderle 2012: 12) unterscheiden lassen. Nach genauer Analyse literarischer Utopien schlägt er „Differenzierungen nach Raum und Zeitutopie, nach Eutopie und Dystopie sowie nach anarchistischer wie archistischer Utopievariante“ (148) vor. 
wie auch mit der Bestimmung der Grenzen dieser Ideen (vgl. Marinčič 2015: 312). Wie Schölderles Analysen zeigen, lassen sich bei dem literarischen Genre der Utopie, dem Morus' Utopia als Prototyp dient, drei Elemente ausmachen: die Kritik aktueller sozialer und politischer Missstände, Entwürfe für deren Lösung und potentielle neue Probleme, die durch die Lösungsversuche hervorgebracht werden könnten. Die Analysen zeigen aber vor allem, dass literarische Utopien lange Zeit nicht als politische Programme, die es zu verwirklichen galt, verstanden wurden, sondern als Gedankenexperimente. Doch Étienne Cabets Idee, seinen utopischen Roman Voyage en Icarie (1840) nicht als Denkübung der Literaturgeschichte zu überlassen, sondern ihn in Realität umzusetzen, kam nicht unvermittelt.

Die Verschiebung im Verständnis der Utopie - vom Gedankenexperiment über einen potentiell realisierbaren Entwurf zum gesellschaftlichen Entwicklungsplan - beginnt im 17. Jahrhundert mit Gerrard Winstanleys Werk The Law of Freedom (1652): „Die Utopie war bis dato ein heiter bis ernst gemeintes Gedankenexperiment, ohne den Versuch, konkrete Handlungsanleitungen zu liefern. Die Frage nach Verwirklichung der Utopie rückt erstmals, und nicht zuletzt unter dem Eindruck revolutionärer Ereignisse, mit Winstanley greifbar in den Mittelpunkt.“ (Schölderle 2012: 88) Ein Jahrhundert später, nachdem die Welt in Umrissen kartographiert und der Glaube an den Fortschritt etabliert war, verschiebt sich die Utopie zur „Uchronie“ (106), d.h. die imaginierte Welt wird nicht mehr in einem gegenwärtigen, aber entfernten und isolierten Raum verortet, sondern in einer zeitlich mehr oder weniger entfernten Zukunft antizipiert. „Mit seinem Roman Das Jahr 2440 projiziert Louis-Sébastien Mercier im Jahr 1771 das fiktive Gemeinwesen in eine ferne Zukunft und verlässt damit erstmals die Dimension der räumlichen Gegenwart." (Schölderle 2012: 104)

Die zweite wichtige Verschiebung in europäischen neuzeitlichen Gesellschaftskonzepten, die sich in Timms Ploetz-Figur niederschlägt, ist die allmähliche „Verabsolutierung des Gleichheitsprinzips“ (Schölderle 2012: 93), deren Anfang Schölderle im Kontext der literarischen Utopien in Gabriel de Foignys 1676 anonym in Genf erschienenem Roman Les Avantures de Jacques Sadeur beobachtet. Die Verabsolutierung des Gleichheitsprinzips muss jedoch im doppelten Sinn verstanden werden. Einerseits bedeutet es ein Streben nach absoluter Gleichheit der Menschen (Foigny z.B. spielt auch mit der Idee der geschlechtlichen Gleichheit), andererseits verdrängt es andere Prinzipien und wird zu dem wichtigsten, wenn nicht gar einzigen, Maß gesellschaftlicher Gerechtigkeit erhoben. Wenn Gleichheit im Motto der französischen Revolution noch von Freiheit und Brüderlichkeit flankiert, also kontextuell als wertvoll und bedeutsam bestimmt wurde, wird sie im 19. Jahrhundert aus dieser Trias herausgelöst und hervorgehoben. Alexis de Tocqueville beobachtet kritisch diesen Prozess und bemerkt auch 
eine Emotionalisierung dieser Konzepte: „On a dit cent fois que nos contemporains avaient un amour bien plus ardent et bien plus tenace pour l'égalité que pour la liberté" (Tocqueville 2012: 453). Auf der intellektuellen Ebene beobachtet er in Amerika, wie sich aus dem Konzept der Gleichheit die Idee einer unendlichen Perfektionierung des Menschen entwickelt (vgl. das Kapitel „Comment l'égalité suggère aux Américains l'idée de la perfectibilité indéfinie de l'homme“; Tocqueville 2012: 411). Er sieht aber auch die Gefahren, die dieser Liebe zur Gleichheit entspringen, denn sie macht die Menschen blind und es kann passieren, dass sie nicht bemerken, wie ihnen, während sie auf sie fixiert sind, die Freiheit entgleitet: „ils veulent l'égalité dans la liberté, et, s'ils ne peuvent l'obtenir, ils la veulent encore dans l'esclavage" (455-456).

Diese zwei Verschiebungen prägen die Figur Alfred Ploetz, von dem Wagner berichtet, er sei in seiner Jugend Kommunist gewesen (vgl. Timm 2017: 141). Von Cabet übernimmt er das Bestreben, Ideen zu verwirklichen, die Realität der Idee anzupassen. Er reist nach Amerika, besucht die Ikarier, um die praktische Umsetzung seiner kommunistischen Ideen zu erlernen. Nach der enttäuschenden Erfahrung, die er dort macht, revidiert er seine Vorstellungen, doch nicht im Sinne der Erkenntnis, dass literarische Utopien einer anderen Ordnung angehören als die Wirklichkeit. Im Gegenteil, er revidiert die zu verwirklichende Idee, indem er soziale und biologistische Ansätze verschränkt und radikalisiert. Biologistische, weil er nicht von der Natur, den Lebewesen selbst ausgeht, sondern von Ideen, wie denn die Lebewesen seien und wie sie sein sollten. Ein mit drei Ausrufezeichen versehener Satz, den er in seinem Brief, einem Bericht aus der Neuen Welt, notiert, fasst es zusammen: „Es muss eine biologische Revolution geben, sie muss die soziale ergänzen!!!“ (242) In der Neuen Welt erkennt er, wie vor ihm schon Tocqueville, die große Bedeutung, die der Gleichheit beigemessen wird, und auch er erkennt, dass die Liebe zur Gleichheit auch Schattenseiten hat: „Die schöne Vorstellung der Gleichheit, die schönste Vorstellung, die erst die menschliche Kultur hervorbringen konnte, wird sich selbst zum Hemmnis, diagnostizierte der Freund.“ (241) Doch während Tocqueville, ein Nachfahr der Aufklärung und der französischen Revolution, ihr Hemmnis in der Verblendung sieht, die zum Freiheitsverlust führen kann, versteht Ploetz, der ein Jahr nach dem Erscheinen von Darwins On the Origin of Species geboren wurde, sie als Behinderung der evolutionären Entwicklung: „Die Natur ist nicht gerecht! Erst die Ungleichheit befördert eine bessere Anpassung an das Vorgefundene.“ (241)

Wagners Bericht über diesen Brief, in dem sich Ploetzs Wende vom Kommunisten zum Rassisten vollzieht, ist in der Mitte des umfangreichen Romans positioniert und erinnert so an den Wendepunkt in der Struktur klassischer Tragödien. Der Denkfehler, den er begeht, lässt sich in diesem Abschnitt 
auch auf der Textebene ablesen, und zwar in einer schlechten Dialektik. Die Idee der Gleichheit entsteht durch die Entwicklung des kulturfähigen Menschen. Sie verhindert aber die biologische Entwicklung. Die biologische Ungleichheit verhindert wiederum die soziale Gleichheit. Die Aufhebung dieser verwirrten Gegensätze erreicht Ploetz, indem er sie auf Biologie reduziert und auf eine höhere Stufe der Evolution projiziert: „Die Gleichheit kann nur durch eine allgemeine Höherentwicklung erreicht werden. Wir müssen diesen Kern, der in Cabets Theorie steckt, herauslösen, ihn in den Mittelpunkt stellen: die Aufzucht eines starken, gesunden, vor allem auch schönen Geschlechts, das sich selbst als stark und schön versteht." (241-242) Dass auf der vermeintlich höheren Stufe die Gleichheit wieder zum Hemmnis wird, dass also seine Argumentation keine Lösung bringt, sondern nur in sich kreist, bemerkt Ploetz nicht.

Die Wiedergabe eines Gesprächs zwischen Ploetz und Wagner, die diesem Bericht über den Bericht folgt, klingt wie eine Fortsetzung eines Gesprächs zwischen Elschner und Gottschalk in Morenga, in dem die Bedeutung des Einzelnen diskutiert wurde:

Man muss ihn von Grund auf ändern.

Wen?

Man muss den Menschen und nicht nur dem Menschen helfen. Man muss Arzt werden und nicht nur dem Einzelnen helfen.

Der Arzt hilft dem Einzelnen.

Das auch. Es muss aber der Menschheit geholfen werden.

So etwas konnte er sagen. Er trug seine Überlegungen mit einem bohrenden Finsterernst vor: Die Kleinheit ist unerträglich, die Kleinheit und Schäbigkeit der Menschen. (Timm 2017: 243)

Die Verachtung des Menschen, des Einzelnen, lässt Elschner und Ploetz als Vertreter einer „universalistisch-utopische[n] Technik“ erscheinen, wie Karl Popper die „Methode des Planens im großen Stil, die utopische Sozialtechnik, die utopische Technik des Umbaus der Gesellschaftsordnung oder die Technik der Ganzheitsplanung“" (Popper 1975: 213-215) bezeichnet und die er als gefährlich erachtet, da sie zu totalitären Regimen führt. Dem gegenüber stellt Popper eine Sozialtechnik, die er „für die einzig rationale“ hält, „die von Fall zu Fall angewendete Sozialtechnik, die Sozialtechnik der Einzelprobleme, die Technik des schrittweisen Umbaus der Gesellschaftsordnung oder die Adhoc-Technik“ (ebd.). Timms Protagonisten Gottschalk und Wagner, also die Gegenspieler von Elschner und Ploetz, sind jedoch nicht Vertreter dieser alternativen „Sozialtechnik der Einzelprobleme“. Es geht nicht um eine einfache literarische Bebilderung der Popperschen Dichotomie. Denn Popper lehnt zwar die Universalisierung als Methode ab, behält aber, da er im Rahmen sozialwis- 
senschaftlicher Überlegungen verbleibt, die Methode der Abstrahierung vom Einzelnen auf „Einzelprobleme“ bei. Die Haltung von Gottschalk und Wagner steht hingegen in der Tradition eines Humanismus, der vom Einzelnen ausgeht und nicht von einem abstrakten, vom Einzelnen losgelösten „Problem“. Die zwei Figurenpaare sind nicht nur Streitende, die zwei Meinungen zu einem Thema haben. Sie sprechen vielmehr zwei verschiedene Sprachen, die aus zwei unterschiedlichen Diskurs- und Denktraditionen hervorgehen und zunehmend inkompatibel werden. ${ }^{6}$ Wagner übernimmt nicht Ploetzs Sprache, spricht nicht von dem oder den Menschen, sondern vom Einzelnen, um sich von einer Verallgemeinerung zu distanzieren, die schon Morus ironisiert hatte, von dem „Menschen im allgemeinen' (...), der doch ein wahrer Koloß ist und größer als jeder Riese" (Morus 2014: 88).

Die Geschichte von Alfred Ploetz, wie sie von Wagner in Timms Roman erzählt wird, hat durchaus Eigenschaften einer Tragödie. Ihr Held, der anfangs Gutes will und später Böses tut, begeht einen tragischen Irrtum, der einen Wendepunkt in der Mitte der Erzählung markiert. Später muss er einsehen, dass die Experimente, denen er viele Jahre seines Lebens widmet, seine These von der degenerativen Wirkung des Alkoholkonsums auf das Erbgut nicht bestätigen. Schließlich schafft er es, sich über ideologische Zerwürfnisse hinwegzusetzen, als er seinem Freund Wagner das Leben rettet, also eben jenem besonderen Einzelnen, der ihn einst an seine Verantwortung dem Einzelnen gegenüber ermahnte. Doch bildet die Geschichte von Ploetz nur einen Erzählstrang im Roman und zu einer katharsis - soweit sie überhaupt das Potential dazu hätte, da es nicht klar wird, ob Ploetz das gesamte Ausmaß seines Fehlers einsieht - führt sie nicht. Erzählt wird sie nämlich in einer Welt, die sich längst mit den Ideen der Rassenhygieniker angefreundet hat und potentielle Gefahren - im Anbahnen des Kalten Kriegs - bereits in anderen Ideologien sucht. Während Hansen, der Protagonist der Rahmengeschichte, den Befehlen der Alliierten gehorchend noch Wagner verhört, um die Verbrechen der Eugeniker aufzudecken und zu verstehen, gilt die Sorge der Amerikaner bereits der potentiellen Gefahr, die von seinen einstigen Sympathien für marxistische Ideen ausgehen könnte (vgl. Timm 2017: 466). Diese Gefahr besteht nicht mehr und Hansens Mission ist beendet. Die Welt hat die Idee der biologischen Perfektionierung des Menschen sowohl von der nationalsozialistischen Ideologie wie auch von den utopischen Bestrebungen nach gesellschaftlicher Gerechtigkeit befreit und sie in die kapitalistische Ideologie überführt, in der sie durch Optimierung der Leistungsfähigkeit im Konkurrenzkampf

6 In dem in Südamerika spielenden Roman Der Schlangenbaum, in dem die Hauptfigur auch Wagner heißt und der Aspekte des Post- und Neokolonialismus thematisiert, trifft die Kritik den Paternalismus der europäischen Intellektuellen aller Disziplinen: „Es sind die ewigen Klugscheißer, auch dann, wenn sie sich selbst als Eurozentristen kritisieren.“ (Timm 2010b: 216) 
am Arbeitsmarkt und in sozialen Selbstinszenierungen der Profitmaximierung dient. Auch wenn diese Idee durch ideologische Rekontextualisierung, die von diversen Ethikkommissionen überwacht wird, zunehmend annehmbar erscheint, bleibt die Grundstruktur des Irrtums gleich: Der Mensch macht sich die Welt untertan, erhebt sich als Richter über sie und befindet sie als unzulänglich. Sie soll seiner Idee angepasst werden, einer Idee, die a priori gut ist, da sie ein Produkt der menschlichen Kultur ist, die aber wiederum verbessert werden kann, da der Mensch als Teil der Welt ebenfalls verbessert werden muss. Diesen selbstverachtenden Gedankenverstrickungen entgegnet Timm nicht mit einer Gegenargumentation, sondern mit literarischen Bildern, deren Träger eine Randfigur ist. George, Hansens Mitbewohner, First Lieutenant und Psychiater, ist Hobby-Ornithologe und macht Hansen auf die Vielfalt und Schönheit der Vögel aufmerksam:

Darin liege das ganze Wunder der Schöpfung. Darwin möge ja recht haben, aber die Möglichkeit dieser Entfaltung, die dann auch das genießende Ohr finde, das sei genau das, was es zu bewahren gelte.

Tell it to your eugenics idiot.

He's dead.

I know, but tell him anyway. (Timm 2017: 170)

Dieses leicht pathetische Plädoyer für das Wahrnehmen und Akzeptieren der Welt, wie sie ist, suggeriert, da es von einem Psychiater geäußert wird, dass die verwirrten, in sich kreisenden Argumentationen der Eugeniker nicht nur Denkfehler, sondern Symptome einer Pathologie sind, und zwar einer Pathologie, der Timm bereits in Morenga auf der Spur war: „Vielleicht ist etwas in ihnen, was ihnen selbst hassenswert ist, ein Teil ungelebten Lebens." (Timm 2010: 388) Doch auf die psychopathologischen Aspekte kann im vorliegenden Artikel, der sich vor allem auf den literarischen Kontext und den philosophischen Utopiediskurs fokussiert, nicht mehr eingegangen werden.

Der knappe, ironische, in Englisch gehaltene Schlagabtausch zwischen Hansen und George, der das Plädoyer abschließt, bricht nicht nur den pathetischen Ton, sondern verweist auch auf die Gegenwart sowie auf die Bedeutung des Erzählens. Trotzdem erzählen, auch wenn scheinbar niemand mehr zuhört, hat nicht nur eine therapeutische, sondern auch eine anthropologische Funktion, wie Jochen Vogt bereits in seinem Kommentar zu Timms Novelle Entdeckung der Currywurst feststellte: „Erzählen heißt am Leben bleiben.“ (Vogt 2014: 406) Gerade weil viele Vogelarten vom Aussterben bedroht sind, sollte man erst recht über die Vielfalt ihrer Gesänge erzählen, nicht nur, um sie zu erhalten, sondern auch das „genießende Ohr", uns selbst. 
Uwe Timms Ikarien ist ein komplexer Roman, dessen Komplexität durch die Verweise auf seine älteren Werke noch gesteigert wird und von jahrzehntelanger tiefgehender Auseinandersetzung mit dem Stoff zeugt. Im vorliegenden Artikel wurde vor allem auf seine Verwandtschaft mit dem Roman Morenga eingegangen, da sie mit der Genese des Romans verbunden ist. Obwohl Timm weit in die Geschichte des 19. und 20. Jahrhundert zurückgreift, sind die Probleme und Denkmuster, die er thematisiert, heute aktuell: sie prägen die Gegenwart und Zukunft des 21. Jahrhunderts. Das Fazit lässt sich nicht auf einfache Statements reduzieren, außer in einer Art übergeordneter Beobachtung, dass die Komplexität des Romans auf die Komplexität der Welt und des in ihr verorteten und sie wahrnehmenden Einzelnen verweist. Diese sollten nicht im Namen eines abstrakten Universalismus reduziert und wegrationalisiert werden. Timms Erzählen leistet Widerstand dagegen und zeigt, dass der Einzelne im 21. Jahrhundert eine Auseinandersetzung mit literarischen Utopien braucht, um die Grenzen seiner Ideen und Denkmuster zu erkunden und die Gegenwart in ihrer Vielfalt wahrzunehmen.

\section{LITERATURVERZEICHNIS}

Durzak, Manfred: „Zweimal Deutsch-Südwestafrika: Uwe Timms Roman Morenga und Gerhard Seyfrieds Roman Herero."In: David Basker (Hg.): Uwe Timm II. Cardiff: University of Wales Press, 2007, S. 37-49.

Frederiksen, Elke: „Writing Across Borders.” In: Jacqueline Vansant, John Pustejovsky, Barbara Becker-Cantarino: ,Wenn sie das Wort Ich gebraucht: Festschrift fur Barbara Becker-Cantarino von FreundInnen, SchülerInnen und KollegInnen. Leiden, Boston: Brill Rodopi, 2013, S. 249-271.

Goethe, Johann Wolfgang: Faust. München: C.H. Beck, 2007.

Marinčič, Marko: „Svetovi brez bogov, svetovi novih bogov“. In: Neda Pagon, Saša Pagon, Neven Borak (Hg.): Utopije - še vedno : zbornik o utopijah v 21. stoletju. Ljubljana: Studia humanitatis, 2015, S. 307-319.

Moritz, Rainer: Uwe Timms Roman zum Rassenwahn: Arbeit an der biologischen Revolution. NZZ, 18.10.2017. <https://www.nzz.ch/feuilleton/ uwe-timms-roman-zum-rassenwahn-arbeit-an-der-biologischen-revolution-ld.1322085>

Morus, Thomas: Utopia. Stuttgart: Reclam, 2014.

Neuhaus, Stefan: „Plötzlich findest du dich in einem Buch wieder, und du bist ein anderer." Grenzauflösungen als konstitutives Merkmal von Uwe Timms Werk. In: Edgar Platen, Martin Todtenhaupt (Hg.): Grenzen, Grenzüberschreitungen, Grenzauflösungen. Zur Darstellung von Zeitgeschichte in deutschsprachiger Gegenwartsliteratur. München: Iudicium, 2004, S. 79-93. 
Otte, Carsten: Tod und Zynismus. Roman „Ikarien“ über NS-Eugeniker. Die Tageszeitung, 10.10.2017. <http://www.taz.de/!5451626>

Popper, Karl R.: Die offene Gesellschaft und ibre Feinde 1. München: Francke, 1975. Schölderle, Thomas: Geschichte der Utopie. Köln, Weimar, Wien: Böhlau Verlag, 2012.

Schöll, Julia: „der Autor, ich’. Auktoriale Selbstentwürfe in Uwe Timms Poetologie(n)“. Text+Kritik, Uwe Timm, Heft 195, Juli 2012, (VII/12), 28-37. Setzwein, Christine: Die zwei Gesichter eines Arztes. Süddeutsche Zeitung, 8. Juli 2018. $<$ https:/www.sueddeutsche.de/muenchen/starnberg/geschichte-die-zwei-gesichter-eines-arztes-1.4045844>

Timm, Uwe: Der Schlangenbaum. München: dtv, 2010(b).

Timm, Uwe: Ikarien. Köln: Kiepenheuer \& Witsch, 2017.

Timm, Uwe: Morenga. München: DTV, 2010.

Tocqueville, Alexis de: De la démocratie en Amérique. Paris: Institut Coppet, 2012.

Vogt, Jochen: Erinnerung, Schuld und Neubeginn. Deutsche Literatur im Schatten von Weltkrieg und Holocaust. Bern: Peter Lang, 2014.

Špela Virant

University of Ljubljana spela.virant@guest.arnes.si

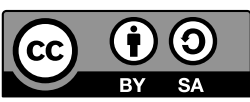

\section{“Tell him anyway": roman Ikarien Uweja Timma}

Roman Ikarien avtorja Uweja Timma je postavljen v Nemčijo tik po drugi svetovni vojni, kjer Wagner mlademu ameriškemu vojaku pripoveduje o življenju evgenika Alfreda Ploetza. Čeprav Timm posega po zgodovinski snovi, je roman aktualen in prej bi ga lahko označili kot filozofskega kot pa zgodovinskega, saj sprašuje po idejah, miselnih vzorcih in napakah, ki posameznika ženejo k nasilju, rasizmu in genocidu. Ta vprašanja Timma zaposlujejo že od njegovih pisateljskih začetkov, zato članek roman Ikarien postavlja $\mathrm{v}$ kontekst njegovega opusa in v kontekst zgodovine utopij, na katere se navezuje že s samim naslovom.

Ključne besede: sodobna nemška književnost, Uwe Timm, Ikarien, Morenga, evgenika, utopija 


\section{"Tell him anyway": The Novel Ikarien by Uwe Timm}

The novel Ikarien (2017) by Uwe Timm is set in Germany after WW II, where Wagner tells the story of the German eugenicist Alfred Ploetz to a young American soldier. Although Timm refers to historical facts about Ploetz and the Icarian movement, the novel deals with philosophical themes relevant to the 21 st century reader, while asking basic questions about the ideas, thought patterns and fallacies that lead to racism, violent behavior, and genocide. Since Timm writes about these themes for decades, the article suggests a reading of the novel in the context of his early work and the history of utopian concepts.

Keywords: modern German literature, Uwe Timm, Ikarien, Morenga, eugenics, utopia 